\title{
Placement privilégié fiscalement
}

\author{
Profitez des avantages d'une prime unique
}

Désirez-vous un placement performant avec un capital garanti de $100 \%$ et des avantages fiscaux? AXA CAPITAL COMFORT, testé et conseillé par nos soins, réalise vos vœux.

Avec AXA CAPITAL COMFORT votre capital est investi dans un fonds orienté en actions Fonds BCV Dynamic Floor Fund World Expoequity REP (CHF). A l'échéance du contrat, la valeur totale de vos parts de fonds est versée, mais au moins la somme garantie à la conclusion du contrat. Vous profitez de cette façon des plus hautes performances, sans devoir renoncer à la sécurité financière de votre placement. De plus pendant l'ensemble de la durée de la police, aucun frais de dépôt, de courtage ou de frais de gestion ne sont perçus.

Si vous vous décidez jusqu'au 30 juin 2006 de conclure un contrat AXA CAPITAL COMFORT, le timbre fédéral de $2,50 \%$ est offert.

Exemple de calcul: homme, âge 52, prime unique 100 000.-

Durée 10 ans Durée 12 ans

Somme minimale garantie en cas de décès :

CHF 131104.- CHF 139077.-

Somme minimale garantie en cas de vie:

CHF 100000.- CHF $100000 .-$

Versement à échéance, libre d'impôts, rendement du fonds $5 \%$ :

CHF 152709.- CHF $167267 .-$

Rentabilité nécessaire d'un placement imposé (taux marginal 35\%):

$6,65 \% \quad 6,74 \%$

Vous gagniez pendant cette période de promotion:

CHF 2500.- CHF 2500.-

\section{Profitez maintenant et commandez votre offre personnelle.}

Vos besoins sont au centre de nos préoccupations. Notre nom vous garantit le conseil professionnel de nos conseillers qui possèdent une longue expérience dans le domaine de l'assurance, des produits bancaires et financiers. Grâce à notre indépendance nous pouvons vous offrir les meilleurs produits adaptés à vos besoins personnels.

\section{Talon réponse}

Prénom/Nom

Adresse

NPA/Lieu

Date de naissance

Téléphone privé/cabinet

Atteignable facilement (heure)

Je désire une offre personnelle.

Je m'intéresse aux produits suivants:

Caisse maladie

3ème pilier lié a

Planification financière

O
Caisse de pension - LPP

Protection juridique prof.

O Responsabilité civile prof.
Roth Gygax \& Partner AG $\mathbf{\square}$ Service de coordination Moosstrasse 2 - 3073 Gümligen

Téléphone 0319595000 \#ax 0319595010 mail@fmhinsurance.ch $\square$ www.fmhinsurance.ch 